\title{
Breath analysis of cancer in the present and the future
}

\author{
Reef Einoch Amor (1) ${ }^{1}$, Morad K. Nakhleh $\mathbb{1}^{2,3}$, Orna Barash ${ }^{1}$ and \\ Hossam Haick ${ }^{1}$
}

Affiliations: ${ }^{1}$ Dept of Chemical Engineering and Russell Berrie Nanotechnology Institute, Technion - Israel Institute of Technology, Haifa, Israel. ${ }^{2}$ Inserm UMR_S 999, Hôpital Marie Lannelongue, Le Plessis-Robinson, France. ${ }^{3}$ University of Paris-Sud, Faculté de Médecine, Université Paris-Saclay, Le Kremlin-Bicêtre, France.

Correspondence: Hossam Haick, Dept of Chemical Engineering and Russell Berrie Nanotechnology Institute, Technion - Israel Institute of Technology, Haifa 3200003, Israel. E-mail: hhossamatechnion.ac.il

@ERSpublications

Breath volatolomics is a highly promising frontier in non-/minimally invasive personalised and evidence-based cancer medicine that could be implemented for a wide variety of cancers in a noninvasive, affordable and easy-to-use manner http://bit.ly/2XeMsDb

Cite this article as: Einoch Amor R, Nakhleh MK, Barash O, et al. Breath analysis of cancer in the present and the future. Eur Respir Rev 2019; 28: 190002 [https://doi.org/10.1183/16000617.0002-2019].

ABSTRACT Most of the currently used diagnostics for cancerous diseases have yet to meet the standards of screening, as they are insufficiently accurate and/or invasive and risky. In this review, we describe the rationale, the progress made to date, and the potential of analysing the exhaled volatile organic compounds as a pathway for enabling early diagnosis of cancer and, therefore, for achieving better clinical prognosis and survival rates. The review highlights the major advancements made in this field, from fundamentals, up to translational phases and clinical trials, with a special emphasis on sensing platforms based on nanomaterials. The prospects for breath analysis in early cancerous disease are presented and discussed.

\section{Introduction}

Cancer is a devastating disease that continues to be faced by numerous challenges, including delayed diagnosis, low efficacy of general anti-cancer therapy and heterogeneity of disease [1]. Unfortunately, for some of the most common imaging techniques currently used to diagnose cancer, the sensitivity depends mostly on tumour size, and the techniques are costly and/or are not amenable to widespread screening because they are time-inefficient [2]. For example, the United States Preventive Services Task Force has shown that the overall sensitivity and specificity of low-dose computed tomography (CT) for lung cancer range from $80 \%$ to $100 \%$ (table 1 ). However, there is still a need to evaluate risks associated with radiation exposure and the relatively high rate of false-positive results [2]. Magnetic resonance imaging and positron emission tomography scans are also used for the detection and staging of cancer. However, their sensitivities and specificities in lung cancer, for example, remain unsatisfactory (table 1). None of these methods can provide accurate cancer staging; thus, complementary invasive procedures such as bronchoscopy, transthoracic needle aspiration and endoscopic ultrasound are needed [25]. Mammography screening, together with breast examination, has reduced mortality from breast cancer by $29 \%$. However, the sensitivity of mammography is highly dependent on the age of the woman, the breast density and breast cancer history, and can be inaccurate in tumours smaller than $1 \mathrm{~mm}[10,11]$.

To date, histological biopsy remains the gold standard diagnostic in cancer, although it is invasive, risky, time-consuming and expensive [26]. Therefore, there is an urgent need for inexpensive and noninvasive diagnostics that would allow: 1) promotion of early detection of cancer; 2) stratification of the population

Provenance: Submitted article, peer reviewed.

Received: Jan 102019 | Accepted after revision: May 192019

Copyright CERS 2019. This article is open access and distributed under the terms of the Creative Commons Attribution Non-Commercial Licence 4.0. 


\begin{tabular}{|c|c|c|c|c|c|c|c|c|c|c|c|c|c|c|}
\hline \multirow[t]{2}{*}{ Cancer } & \multirow[t]{2}{*}{ Method } & \multirow[t]{2}{*}{ Ref. } & \multicolumn{3}{|c|}{ Differential diagnosis } & \multicolumn{3}{|c|}{ Diagnosis } & \multicolumn{3}{|c|}{ Monitoring } & \multicolumn{3}{|c|}{ Classification/staging } \\
\hline & & & Sens. & Spec. & Acc. & Sens. & Spec. & Acc. & Sens. & Spec & Acc. & Sens. & Spec. & Acc. \\
\hline \multirow[t]{2}{*}{ Lung } & Low-dose CT & {$[2,3]$} & $80-100$ & $>90$ & & $52-65$ & $48-79$ & PPV 100 & & & & $52-91$ & $48-95$ & $\begin{array}{l}\text { PPV 79; } \\
\text { NPV 78-93 }\end{array}$ \\
\hline & Breath analysis & [4-9] & $60-100$ & $57-100$ & $59-94$ & $70-91$ & $73-88$ & 88 & $28-93$ & $75-100$ & $80-92$ & $78-92$ & $73-95$ & $83-88$ \\
\hline \multirow[t]{2}{*}{ Breast } & Mammography & {$[10,11]$} & $93-96$ & $89-93$ & $71-87$ & $42-88$ & $75-92$ & 70 & & & & & & \\
\hline & Breath analysis & {$[12,13]$} & $63-94$ & $76-82$ & $71-88$ & & & & & & & $71-100$ & $70-100$ & $71-100$ \\
\hline \multirow[t]{2}{*}{ Ovarian } & CA125 and TVU & [14] & $77-89$ & $75-99$ & & & & & & & & & & \\
\hline & Breath analysis & {$[15,16]$} & & & & $59-83$ & $61-100$ & $61-89$ & & & & & & \\
\hline \multirow[t]{2}{*}{ Gastric } & Gastroscopy & [17] & & & & $69-84$ & $85-96$ & & & & & & & \\
\hline & Breath analysis & [18-21] & $71-97$ & $87-91$ & $77-96$ & $73-96$ & 98 & $92-95$ & & & & $45-93$ & $41-98$ & 43-92 \\
\hline \multirow[t]{2}{*}{ Colorectal } & Colonoscopy & {$[22,23]$} & $66-99$ & $30-100$ & $70-96$ & $88-98$ & $82-99$ & & & & & & & \\
\hline & Breath analysis & [24] & & & & 85 & 94 & 91 & & & & 100 & 88 & 94 \\
\hline
\end{tabular}


based on their bio-specification for personalised therapy; and 3) fast assessment of treatment efficacy at the bedside, to change the therapeutic approach accordingly.

To meet these challenges, intensive effort has been invested in the last two decades to reach new biomarker-based platforms for novel diagnostics and functional genomic analysis that could be broadly applicable in a wide range of cancers. While several cancer-related biomarker platforms have shown improvements in clinical outcomes and survival rates, they suffer from significant limitations for clinical practice [27]. Most of these biomarkers are organ-specific and thus might not be informative enough [27].

A highly promising development in cancer biomarkers is based on volatolomics, i.e. on analysis of volatile organic compounds (VOCs) (semi-volatile and highly volatile) that emanate from cancer cells and/or their micro-environment and that are detectable in different body fluids (exhaled breath, blood, urine, sweat, etc.) depending on their tissue/blood and blood/air partition coefficients [28]. The rationale behind this approach rests on the fact that, during pathophysiological processes, alterations in cell metabolism lead to shifts in the production of VOCs, which are by-products of biochemical reactions [29]. Hypoxia, hyperproliferation of cells, excessive inflammatory and reactive oxygen species activity, and other cancer-related pathological mechanisms result in marked shifts in the spectra and concentrations of VOCs, both locally and systematically [30].

VOCs in exhaled breath have been correlated to the pathophysiology of cancers [28, 31, 32]. It is reasonable to hypothesise that such shifts could be detectable as soon as the cancer is established. Additionally, as a VOC profile reflects cancer-related metabolism [33, 34], the shifts may be informative in several other ways, including giving some indication of specific genetic mutations that might be responsive to treatment and monitoring [30].

For these reasons, breath analysis could be the basis of a noninvasive method for the early diagnosis of cancer. The particularly significant feature that can be exploited is that each disease has its own unique volatolome pattern; therefore, the presence of one disease would not necessarily screen out others [35]. This review critically examines the progress made so far in the field of breath analysis, mainly as a clinical tool. Future perspectives, as well as challenges in this field, are discussed.

\section{Evidence for the "omics" of breath testing}

In the last two decades, extensive work has been carried out on various aspects of breath analysis for cancer detection, from fundamental biological to translational research and development studies.

On the basic science front, a variety of spectroscopic and spectrometric techniques have been used to carry out in vivo, in vitro and ex vivo experiments to gain knowledge about VOC biomarkers. These methods have provided hundreds of tentative identifications of the VOCs emitted directly by cells and tissues in culture, independently of the systemic alterations that might be due to secondary mechanisms and haemodynamics $[29,36]$. For example, VOCs produced by three types of primary lung cancer cells have been compared with those in the breath of cancer patients. Of five VOCs that discriminated cancer patients from healthy controls using breath samples, one was shared with lung cancer cells, suggesting a potential biomarker [37]. Another study compared VOC composition of the lung cancer cell line A549 and that of urine from mice implanted with the same cells. Seven VOCs proved to be significantly higher compared to normal cancer cell lines and urine from normal mice [38].

The source of the VOCs and semi-volatile compounds is mainly hypothetical and relies mostly on endogenous and exogenous processes. Disease-specific VOCs are assumed to be produced mainly through changes in specific biochemical pathways in the body, primarily related to oxidative stress, cytochrome P450, liver enzymes, and carbohydrate and lipid metabolism. Most exogenous VOCs and semi-volatile compounds are related to smoking habits, air pollution, etc.

VOCs have been related to a multitude of clinical conditions; as such, it is unlikely that a single VOC biomarker can characterise a disease $[28,29,33,36]$. It has more often been shown that cancers change the patterns of VOCs, which are altered according to the type, stage and subcategory of the cancer [31]. Also involved are VOCs that originate from purely endogenous processes (e.g. isoprene), VOCs that originate from either endogenous or exogenous sources (e.g. acetone), and VOCs that originate from exogenous processes alone (e.g. toluene) [29]. The vast majority of VOCs related to cancer patients are from alkanes, alcohols, aldehydes, ketones, nitriles and aromatic compounds [29]. Although their source is still speculative, several possible biochemical pathways have been suggested. For example, oxidative stress in the micro-environment of a cancer, due to hypoxic and/or inflammatory conditions, contributes to the formation of alkanes and methylated alkanes [30]. Over-activation of cytochrome P450 enzymes in cancer patients might elevate alcohol levels. Along with these enzymes, cancer is also characterised by raised alcohol dehydrogenase and aldehyde dehydrogenase, which are responsible for aldehyde catabolism and 
metabolism, respectively $[29,34]$. Excessive cell proliferation resulting from local hypoxia leads to anaerobic respiration in which the glycolytic pathway of energy production releases ketones and alcohols [30]. In addition to these biomarkers, some exogenous VOCs have also been found at increased levels in cancer patients, among them benzene, toluene and styrene. The source of these highly volatile pollutants could be intake/absorption throughout the gastrointestinal system and/or skin, in addition to direct inhalation. It remains unclear, however, whether these VOCs could be used as biomarkers, or have the potential to indicate the initiation/progression of diseases, mainly because they occur in the exhaled breath of healthy subjects [29].

Thorough review of the literature in the field of breath analysis shows that specific VOCs have been suggested as biomarkers in several different types of cancer. For example, decane increased in hepatic cancer [39] and lung cancer [29], indicating that different cancers might also share part of the spectrum of VOC biomarkers. In lung cancer, exhaled breath partially overlaps with the VOCs from cell lines [29]. This finding indicates that, in addition to the VOCs that are directly emitted by the cancerous cells, other VOC biomarkers can be found in exhaled breath due to secondary and/or systemic mechanisms accompanying the disease itself [30].

NAKHLEH et al. [35] analysed breath samples from 1404 subjects, including 813 patients diagnosed with one of 17 different diseases, and 591 control subjects. Over 400 of the patients were diagnosed with eight different cancers. Quantitative gas chromatography linked with mass spectrometry analysis was used to assess the similarity and/or differences in exhaled breath composition, not only compared to healthy controls but also when compared between the different diseases. The results showed that each of the diseases has its own unique VOC molecular print and, therefore, the presence of one cancer would not screen out others. Statistical analysis indicated that none of the studied VOCs could serve as a stand-alone biomarker for cancerous diseases. However, a pattern of 13 VOCs helped to discriminate between these diseases. These results support the hypothesis that, due to the similarities in the pathogenic mechanisms (hyperproliferation, local hypoxia, oxidative stress, etc.) involved in different cancers, the altered VOCs were common to the majority of cancers, yet represented an altered balance between them, leading to the discovery of "unique" signatures for each disease. These results were supported by hierarchical clustering, showing in most cases that cancerous conditions were naturally clustered together, giving close similarities in their VOC profiles [35]. These results are in agreement with those of PENG et al. [40], who showed four discriminative volatolomic signatures identified in 96 patients diagnosed with lung, colon, breast or prostate cancer. Analysis of exhaled breath in multiple morbidities remains narrow.

\section{Determining the composition of the cancer volatolome}

Two key analytical approaches have been established to assess VOCs in exhaled breath. The first relies on an analytical chemistry approach using chromatography with or without two spectrometric techniques that aim to identify and quantify the chemical nature and composition of VOCs in any given sample. This approach enables one to explore each compound disjointedly and estimate the probability that any quantifiable compound could become a biomarker of disease. The second approach is inspired by the mammalian sense of smell, where semi-selective/cross-reactive sensors combined with pattern recognition methods are adopted. This approach can identify patterns of VOCs that are like "fingerprints" of a disease. This approach cannot identify each VOC, but it can reveal the overall pattern and, thus, can be used in future to pinpoint similar cases [41].

To date, several chemical sensors for the assessment of VOCs have been developed, which are now being tested as cancer-diagnostic platforms. Each of these platforms has its unique feature and different affinities to spectra of VOCs. Chemical sensors based on metal oxides, for example, have a high affinity for negatively charged oxygen; they are broadly used in chemiresistors as a sensing layer for oxidising or reducing gases [42]. Colorimetric sensors are based on chemo-responsive indicators that react chemically and change their colour upon exposure to different chemical species. This change in colour is used as a value of the measurement [43]. Surface acoustic wave sensors are based on frequency modulation of waves travelling along the surface of an elastic substrate. The sensing mechanism is based on a frequency shift of the sensing layer, which can be caused by changes in conductivity, stress effect, mass loading and viscoelastic effect as a result of gas absorption [44]. Chemiresistors based on chemically modified (monolayer-coated) metal nanoparticles or single-walled carbon nanotubes (SWCNTs) have shown valuable assets in terms of medical use, including: large surface-to-volume ratio, low-output impedance, fast response and recovery times, tolerance to humidity and low-voltage operation [45]. Additional types of available gas sensors for breath analysis include optical sensors, field effect transistors based on silicon nanowires, and sensors based on the piezoelectric transduction mechanism (figure 1 gives simplified working mechanisms). The potential for sensor-based breath analysis to be a routine medical test is further increased due to it being easy and inexpensive for high-volume 


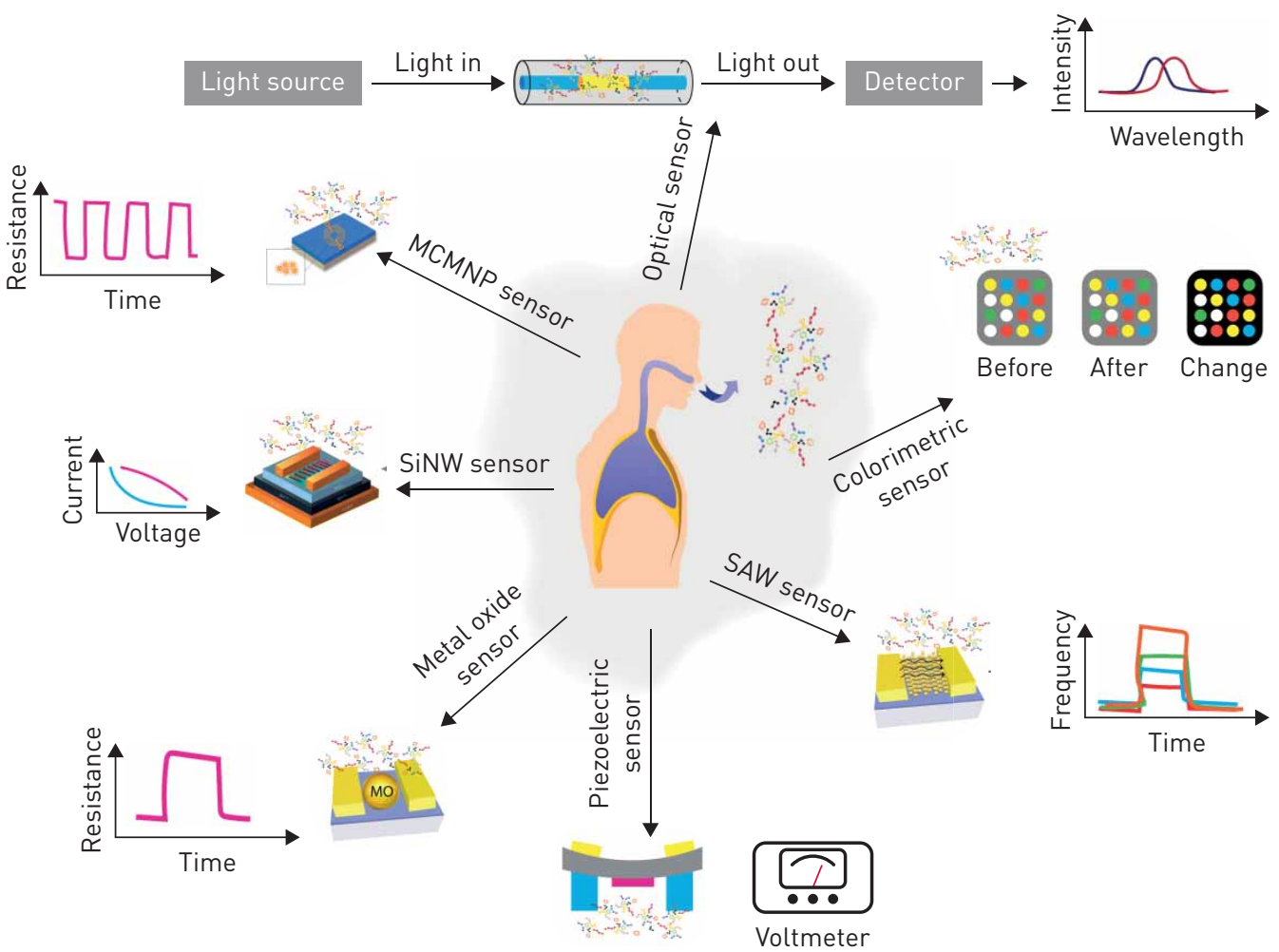

FIGURE 1 Nanomaterial-based sensors as a volatolomic analytical platform: simplified working mechanisms. These sensors may be used in medical care in future volatolome-based diagnostic devices. Clockwise: optical sensors, colorimetric sensors, surface acoustic wave (SAW) sensors, piezoelectric sensors, metal oxide (MO) sensors, silicon nanowire (SiNW) sensors and monolayer-coated metal nanoparticle (MCMNP) sensors.

manufacturing and operation. A detailed review on all types of sensors for VOC-related clinical analysis can be found in BROZA et al. [41].

\section{Chemical sensors for breath analysis of cancer}

Sensor arrays based on chemiresistors using gold nanoparticles (GNPs) and/or SWCNTs have been the most examined sensing technologies for cancer detection by breath sampling. Using these sensor arrays, PENG et al. [40] and NAKHLeH et al. [35] showed their use in detecting and classifying many types of cancer, opening a window for developing a multi-applicable sensor technology for clinical practice. Figure 2a shows a graphical representation of pattern recognition classifiers obtained from the multiple binary volatolomic comparisons of 17 diseases [35]. As seen in the figure, among the cancerous diseases, classification accuracy ranged between $64 \%$ (bladder versus gastric) and 100\% (in several other comparisons). High accuracy was also achieved in discrimination between dependent and clinically challenging cases, for example between colorectal cancer and Crohn's disease or ulcerative colitis, with accuracy of $79 \%$ and $93 \%$, respectively. Lung cancer cases were successfully discriminated from pulmonary arterial hypertension cases with $86 \%$ accuracy, the same as the overall accuracy of all disease-related volatolomic classifiers. Similar statistical analysis of 591 control subjects enrolled in parallel to the patient groups gave $58 \%$ accuracy in classification, indicating that, although intra-individual volatolomic differences occur in the healthy population, shifts during disease states are more dominant and recognisable. In the same study, NAKHLEH et al. [35] found that VOCs were affected by confounding factors, mainly active smoking and age, and that stratifying techniques could be used to correct these effects and eliminate the possibility of biased results. These results show the ability of breath analysis in general, and the use of sensor technologies for breath tests in disease detection and classification. Assessing the utility of these sensors and related findings in the clinic, however, requires further validation in larger and multicentre clinical studies.

Besides the reported ability to discriminate between cancer and control groups, GNP- and SWCNT-based sensors have been used to recognise and distinguish between subcategories of the disease. Table 1 lists the clinical performance of selected examples of breath analysis in cancer compared to the usual diagnostic techniques in the clinics. SHLOmi et al. [4] reported on detection and discrimination between patients with early stage lung cancer and benign lung nodules (figure $2 \mathrm{~b}$ ), as well as on the ability to monitor the 
a)

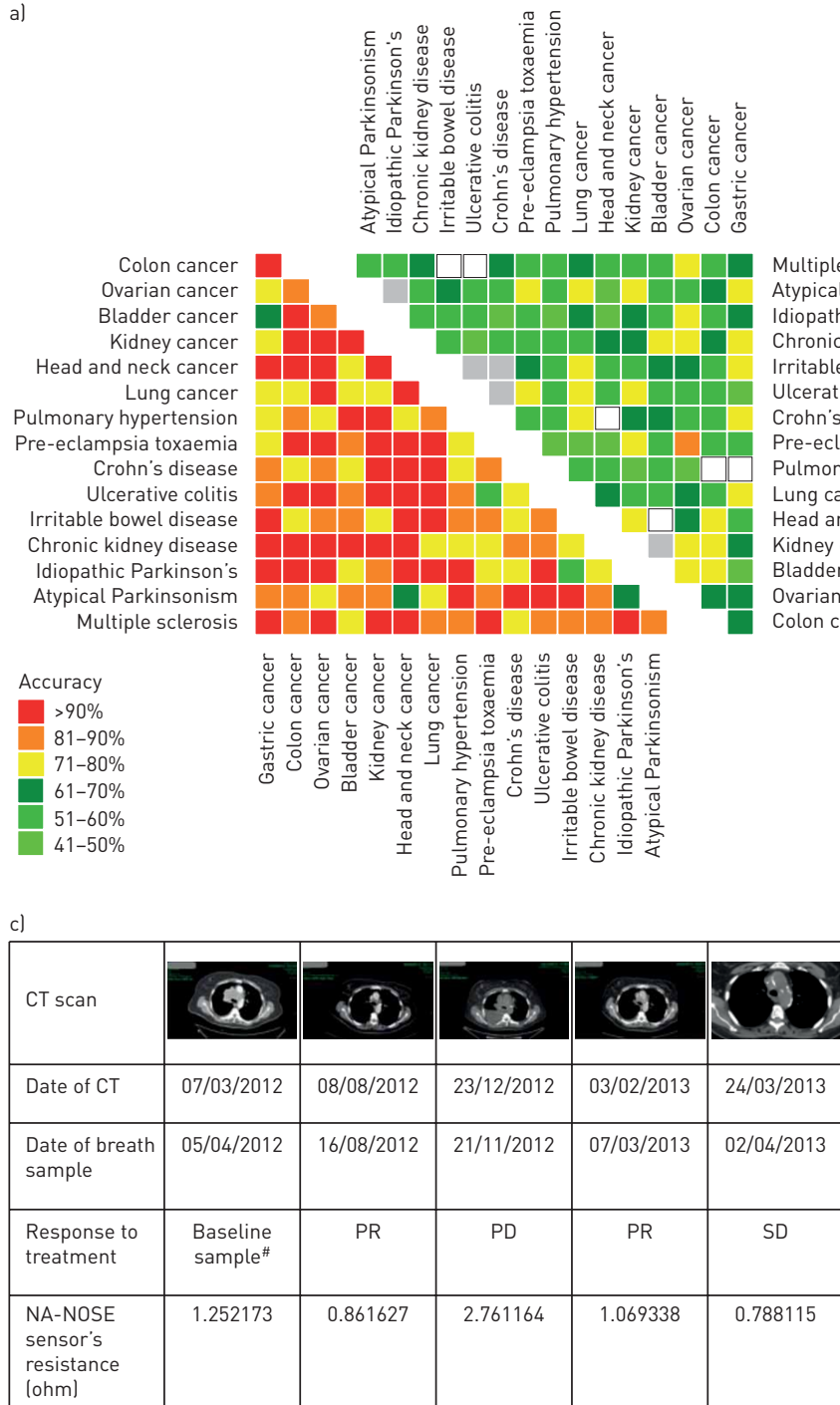

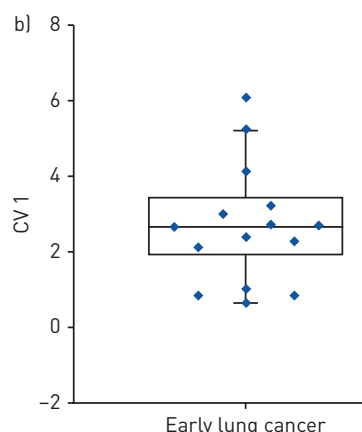

$p<0.0015$

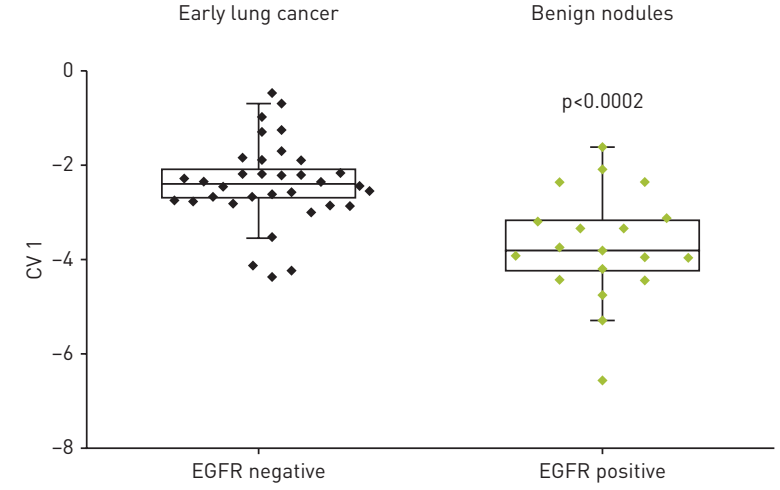

d)
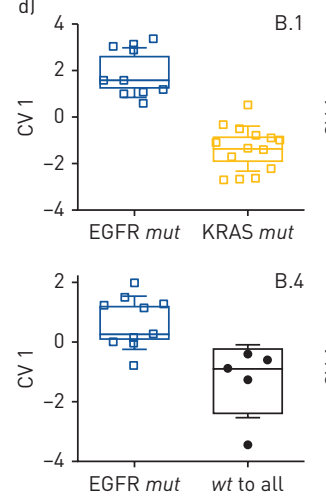

B.1
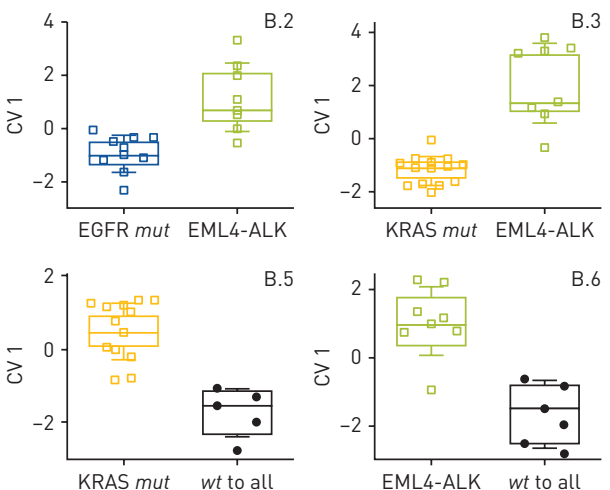

B. 3

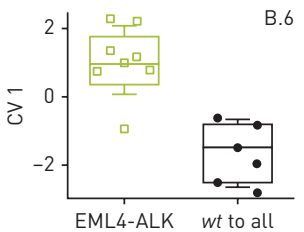

FIGURE 2 Volatolomics in cancer. a) Discrimination between different cancers: graphical representation for the accuracies calculated of binary discriminant factor analysis (DFA) classifiers for disease classification by breath analysis. The left heat map gives comparisons between patients from each group, including eight cancers, resulting in an average of $86 \%$ accuracy. The right heat map represents a comparison of healthy volunteers from each group, resulting in a 58\% accuracy. Reproduced and modified from [35] with permission. b) Cancer histology classification and staging: graphical representation of canonical variable (CV) values calculated from the responses of the sensor array to breath samples taken from lung cancer patients at early stages of the disease compared with benign nodules. A volatolomic signature was also calculated for the discrimination between lung cancer patients harbouring the epidermal growth factor receptor (EGFR) mutation and those without. Reproduced and modified from [4] with permission. c) Cancer monitoring: computed tomography (CT) scans from a lung cancer patient undergoing CT follow-up while receiving chemotherapy. Dates of CT scans, as well as of breath samples, are indicated. The corresponding sensor response in ohms is indicated with relation to each CT scan. PR: partial response; PD: progressive disease; SD: stable disease. ${ }^{\#}$ : before initiation of treatment. Reproduced and modified from [5] with permission. d) Genetic mutations in cancer: DFA maps calculated from signals obtained from sensor arrays to VOCs in the headspace samples of cell lines bearing different genetic mutations associated with lung cancer (EGFR, KRAS and echinoderm microtubule-associated protein-like 4 (EML4)-anaplastic lymphoma kinase (ALK) fusion). wt: wild type. Reproduced and modified from [46] with permission.

response to therapy by analysing VOCs in the breath. The same group also made links between VOC findings and CT scan imaging (figure 2c) [5]. They also reported on the ability to use GNP- and SWCNT-based sensors for the detection and monitoring of patients with the epidermal growth factor receptor (EGFR) mutation (figure 2b). These findings were supported by several in vitro analyses of VOC-related genetic mutations of lung cancer (including EGFR, KRAS and echinoderm microtubule-associated protein-like 4 (EML4)-anaplastic lymphoma kinase (ALK) fusion) [46-51].

\section{Potential and limitations}

Chemical sensors for breath analysis have the potential to be integrated into routine medical testing (illustrated in figure 3). Connected to healthcare providers through "internet of things", an infinite flow of 
VOC information can be collected into a cloud, while constantly re-analysing the data through branched and complex algorithms. This could help in the early detection of cancer and immediate monitoring of disease development. However, there are several limitations impeding this technology from advancing from proof of concept to the practical clinical application. In part, these are due to the (controversial) effects of potential confounding factors in breath analysis, e.g. age, diet, genetics, smoking habits, etc. Since the profile of exhaled breath is instantly modified as a consequence of changes in blood chemistry, the question is raised regarding the timing and method of sampling [34]. It follows that a standardised protocol for uniform and repeatable breath sampling is highly necessary regarding the reliability of the final product. This would require an intensive fundamental study of large groups of healthy volunteers to explore the consistency of breath composition seen under different conditions so that the optimal ones can be selected for future application. Many of these aspects have now been raised and discussed by the European Respiratory Society Task Force [52].

Other challenges in the field of breath analysis of cancer stem from the technical aspects of the sensing technologies and their adaptability to real clinical conditions. For example, almost all reported studies have used a "sterile" environment during analysis, due to the sensors' sensitivity to temperature and humidity levels during measurement. In most studies, techniques like dehumidification, filtering and pre-concentrations have been used to enhance sensor selectivity and response [53]. The choice of statistical method to analyse the sensors' input data is important for future technological applications. Common problems include bias, incorrect sample size (mainly in relation to the number of variables and to the required statistical power, specificity and sensitivity, to prove that a biomarker or a pattern of biomarkers is discriminant), excessive false discovery/over-optimism rates owing to multiple hypotheses being tested,

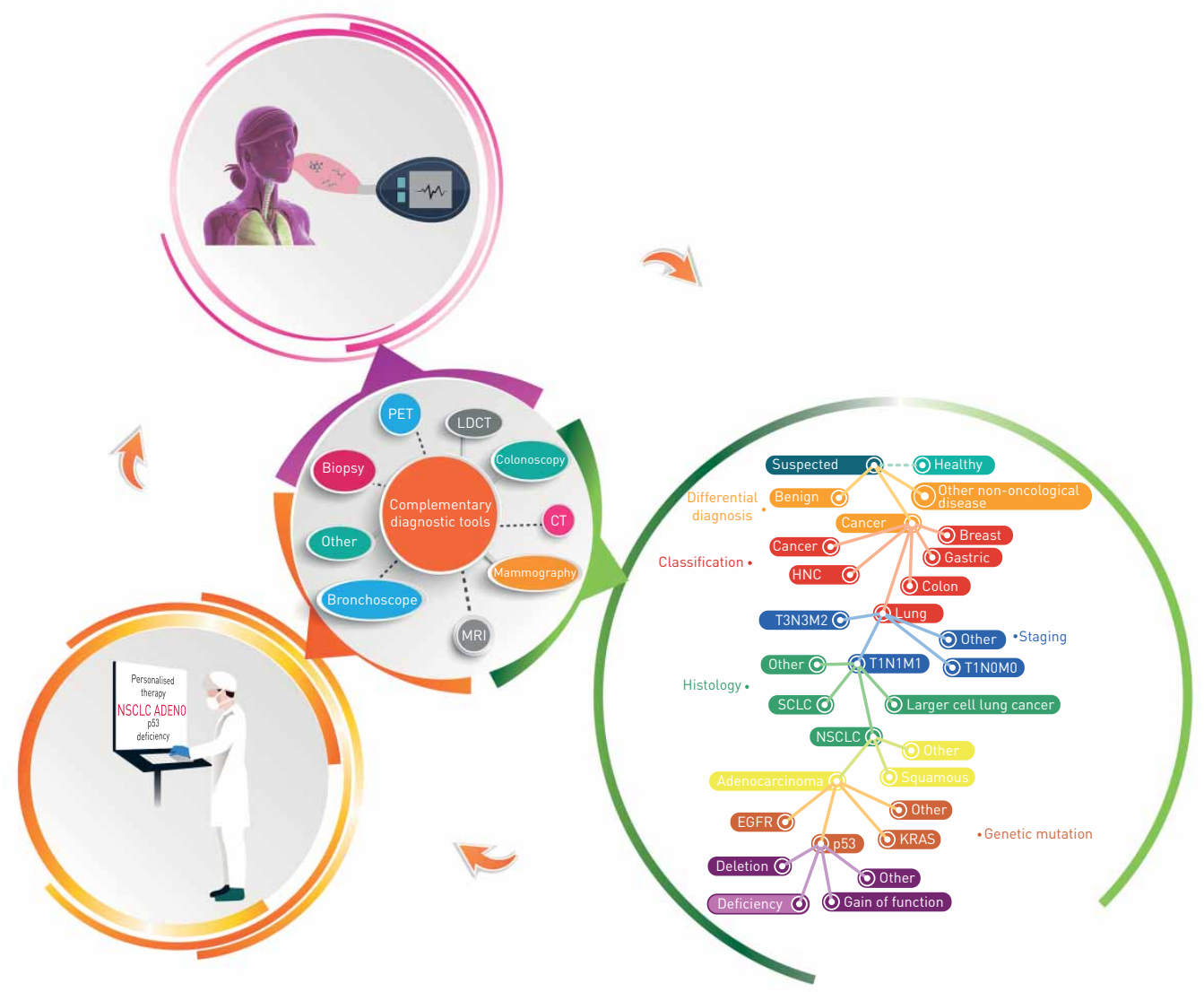

FIGURE 3 Potential of breath analysis as a complementary diagnostic tool in cancer. This is an illustration of the potential of breath analysis and its implementation as a dependent or complementary test that could be beneficial for cancer management. Noninvasive and easy to perform, volatolome assessment could promote early and differential diagnosis, cancer classification, staging and monitoring. However, extensive research and development are required to increase both clinical performance and integration into real clinical-world conditions. PET: positron emission tomography; CT: computed tomography; LDCT: low-dose CT; MRI: magnetic resonance imaging; HNC: head and neck cancer; SCLC: small cell lung cancer; NSCLC: nonsmall cell lung cancer; EGFR: epidermal growth factor receptor. 
wrong choice of numerical methods, inadequate validation and cross-validation, and overcomplicated statistical modelling [54]. Therefore, model development, specification, performance and update transparency is essential. Following these constraints, investigators might strengthen or weaken the model by implementing it on their data or overestimating its credibility.

An additional challenge for breath analysis stems from its inability to provide validated and strong predictive values for both cancer diagnosis, screening and monitoring. Diagnosing cancer with satisfactory sensitivities and specificities that spare most benign lesions is challenging, especially when performed in real time and in vivo. Breath analysis must be capable of providing sufficient sensitivity with maximal negative predictive value. For example, in lung cancer, low-dose CT followed by bronchoscopy is still the gold standard procedure in the diagnosis and monitoring process, despite high numbers of false-positive cases $[2,3]$. Sensor technology for breath analysis may solve this problem and reduce the number of false positives [55].

Readers should keep in mind that all studies mentioned in the current review are still in the small-size, cross-sectional and proof-of-concept stage. Unfortunately, to date, there are no available data to compare between the gold standard diagnostics/screening/monitoring tests and results of validation studies in breath analysis. The papers used in this review are given as representative examples for the potential of breath analysis to contribute in cancer medicine, rather than presenting breath analysis as a tool ready to be used. Comparisons between clinical capabilities of breath analysis studies and gold standard procedures are given in table 1. For example, the prevalence of malignancy in the lung cancer high-risk patient population is $1.5 \%$. Therefore, to identify one cancer case for every $10 \mathrm{CT}$ scans performed, the specificity would need to reach between $85 \%$ and $90 \%$, while the sensitivity of the test must approach $95 \%$ to guarantee that only one out of every 1000 breath-test-negative patients has lung cancer. The same compromises between sensitivity and specificity must be taken in each clinical choice. For diagnosis purposes, for instance, we will want to ensure test accuracy for taking clinical decisions such as nodule resection. Resection will be performed if malignancy probability is $>90 \%$ and follow-up if the probability is $<10 \%$ [56]. For breath analysis to become a real-life clinical tool, clinical standards must be decided by physicians and chemically and clinically validated in large-scale, multicentre studies. For each cancer type, a sufficient cut-off for negative predictive value and positive predictive value must be decided and tested comparing to known clinical standards.

\section{Concluding remarks}

Early detection of diseases, particularly cancer, could increase the 5-year survival rate by three- to five-fold. The attractiveness of breath analysis lies in it being noninvasive, its ease of use, its ability to help prognosis, and its low cost. If not as a stand-alone diagnostic, then it ought to be combined with current methods; it could be a complementary method for increasing the accuracy of detection and giving an earlier diagnosis. However, for breath testing to be developed toward present clinical applications, the abovementioned challenges must be met such that more satisfying clinical performance should first be achieved in terms of sensitivity and repeatability. This might be accomplished through implementing the following main recommendations: 1) better understanding of the origin and pathways of breath VOCs; 2) determination of a universal breath biopsy method that would lead to repeatability; 3) adaptation of sensor-based analysis platforms to clinical-world conditions; and 4) large-scale, multicentre clinical trials with blind validation to confirm the proof-of-concept studies. Global biomedical guidelines for diagnosis should be adopted in the field of breath analysis regarding both research plan and reporting of data. Published papers should stress critical points so that researchers can evaluate the evidence with reliability. This includes detailed and transparent reporting of key study details, from the background and objectives, through methods of collection, measurement and analysis, to model development, performance and decision making $[57,58]$. The development of best clinical practice in the development of volatolomic tests should bring this exciting field to fulfilment.

Conflict of interest: None declared.

\section{References}

1 Lawrence MS, Stojanov P, Polak P, et al. Mutational heterogeneity in cancer and the search for new cancer-associated genes. Nature 2013; 499: 214-218.

2 Humphrey LL, Deffebach M, Pappas M, et al. Screening for lung cancer with low-dose computed tomography: a systematic review to update the US Preventive Services Task Force recommendation. Ann Intern Med 2013; 159: 411-420.

3 Toyoda Y, Nakayama T, Kusunoki Y, et al. Sensitivity and specificity of lung cancer screening using chest low-dose computed tomography. Br J Cancer 2008; 98: 1602-1607.

4 Shlomi D, Abud M, Liran O, et al. Detection of lung cancer and EGFR mutation by electronic nose system. J Thorac Oncol 2017; 12: 1544-1551. 
5 Nardi-Agmon I, Abud-Hawa M, Liran O, et al. Exhaled breath analysis for monitoring response to treatment in advanced lung cancer. $J$ Thorac Oncol 2016; 11: 827-837.

6 Peled N, Hakim M, Bunn PA Jr, et al. Non-invasive breath analysis of pulmonary nodules. J Thorac Oncol 2012 7: $1528-1533$.

7 Broza YY, Kremer R, Tisch U, et al. A nanomaterial-based breath test for short-term follow-up after lung tumor resection. Nanomedicine 2013; 9: 15-21.

8 Mazzone PJ, Wang XF, Xu Y, et al. Exhaled breath analysis with a colorimetric sensor array for the identification and characterization of lung cancer. J Thorac Oncol 2012; 7: 137-142.

9 D’Amico A, Pennazza G, Santonico M, et al. An investigation on electronic nose diagnosis of lung cancer. Lung Cancer 2010; 68: 170-176.

10 Ohuchi N, Suzuki A, Sobue T, et al. Sensitivity and specificity of mammography and adjunctive ultrasonography to screen for breast cancer in the Japan Strategic Anti-cancer Randomized Trial (J-START): a randomised controlled trial. Lancet 2016; 387: 341-348.

11 Sprague BL, Arao RF, Miglioretti DL, et al. National performance benchmarks for modern diagnostic digital mammography: update from the Breast Cancer Surveillance Consortium. Radiology 2017; 283: 59-69.

12 Shuster G, Gallimidi Z, Reiss AH, et al. Classification of breast cancer precursors through exhaled breath. Breast Cancer Res Treat 2011; 126: 791-796.

13 Barash O, Zhang W, Halpern JM, et al. Differentiation between genetic mutations of breast cancer by breath volatolomics. Oncotarget 2015; 6: 44864-44876.

14 Menon U, Griffin M, Gentry-Maharaj A. Ovarian cancer screening - current status, future directions. Gynecol Oncol 2014; 132: 490-495.

15 Kahn N, Lavie O, Paz M, et al. Dynamic nanoparticle-based flexible sensors: diagnosis of ovarian carcinoma from exhaled breath. Nano Lett 2015; 15: 7023-7028.

16 Amal H, Shi DY, Ionescu R, et al. Assessment of ovarian cancer conditions from exhaled breath. Int J Cancer 2015; 136: E614-E622.

17 Choi KS, Suh M. Screening for gastric cancer: the usefulness of endoscopy. Clin Endosc 2014; 47: 490-496.

18 Amal H, Leja M, Funka K, et al. Detection of precancerous gastric lesions and gastric cancer through exhaled breath. Gut 2016; 65: 400-407.

19 Shehada N, Brönstrup G, Funka K, et al. Ultrasensitive silicon nanowire for real-world gas sensing: noninvasive diagnosis of cancer from breath volatolome. Nano Lett 2015; 15: 1288-1295.

20 Shehada N, Cancilla JC, Torrecilla JS, et al. Silicon nanowire sensors enable diagnosis of patients via exhaled breath. ACS Nano 2016; 10: 7047-7057.

$21 \mathrm{Xu} \mathrm{ZQ}$, Broza YY, Ionsecu R, et al. A nanomaterial-based breath test for distinguishing gastric cancer from benign gastric conditions. Br J Cancer 2013; 108: 941-950.

22 Lin JS, Piper MA, Perdue LA, et al. Screening for colorectal cancer: updated evidence report and systematic review for the US Preventive Services Task Force. JAMA 2016; 315: 2576-2594.

23 Schachschal G, Mayr M, Treszl A, et al. Endoscopic versus histological characterisation of polyps during screening colonoscopy. Gut 2014; 63: 458-465.

24 Amal H, Leja M, Funka K, et al. Breath testing as potential colorectal cancer screening tool. Int J Cancer 2016; 138: 229-236.

25 Sihoe AD, Yim AP. Lung cancer staging. J Surg Res 2004; 117: 92-106

26 King JD, Casavant BP, Lang JM. Rapid translation of circulating tumor cell biomarkers into clinical practice: technology development, clinical needs and regulatory requirements. Lab Chip 2014; 14: 24-31.

27 Walt DR, Chiu D, Kuperwasser C, et al. Development of Technologies for Early Detection and Stratification of Breast Cancer. Boston, Tufts University, 2016. Available from: https://apps.dtic.mil/dtic/tr/fulltext/u2/1030618.pdf

28 Broza YY, Zuri L, Haick H. Combined volatolomics for monitoring of human body chemistry. Sci Rep 2014; 4: 4611.

29 Hakim M, Broza YY, Barash O, et al. Volatile organic compounds of lung cancer and possible biochemica pathways. Chem Rev 2012; 112: 5949-5966.

30 Nakhleh MK, Haick H, Humbert M, et al. Volatolomics of breath as an emerging frontier in pulmonary arteria hypertension. Eur Respir J 2017; 49: 1601897.

31 Schmidt K, Podmore I. Current challenges in volatile organic compounds analysis as potential biomarkers of cancer. J Biomark 2015; 2015: 981458.

32 Amann A, de Lacy Costello B, Miekisch W, et al. The human volatilome: volatile organic compounds (VOCs) in exhaled breath, skin emanations, urine, feces and saliva. J Breath Res 2014; 8: 034001.

33 van der Schee MP, Paff T, Brinkman P, et al. Breathomics in lung disease. Chest 2015; 147: $224-231$.

34 Haick H, Broza YY, Mochalski P, et al. Assessment, origin, and implementation of breath volatile cancer markers. Chem Soc Rev 2014; 43: 1423-1449.

35 Nakhleh MK, Amal H, Jeries R, et al. Diagnosis and classification of 17 diseases from 1404 subjects via pattern analysis of exhaled molecules. ACS Nano 2017; 11: 112-125.

36 Buljubasic F, Buchbauer G. The scent of human diseases: a review on specific volatile organic compounds as diagnostic biomarkers. Flavour Fragr J 2015; 30: 5-25.

$37 \mathrm{Yu}$ J, Wang D, Wang L, et al. Detection of lung cancer with volatile organic biomarkers in exhaled breath and lung cancer cells. AIP Conference Proceedings 2009; 1137: 198.

38 Hanai Y, Shimono K, Oka H, et al. Analysis of volatile organic compounds released from human lung cancer cells and from the urine of tumor-bearing mice. Cancer Cell Int 2012; 12: 7.

39 Qin T, Liu H, Song Q, et al. The screening of volatile markers for hepatocellular carcinoma. Cancer Epidemiol Biomarkers Prev 2010; 19: 2247-2253.

40 Peng G, Hakim M, Broza YY, et al. Detection of lung, breast, colorectal, and prostate cancers from exhaled breath using a single array of nanosensors. Br J Cancer 2010; 103: 542-551.

41 Broza YY, Vishinkin R, Barash O, et al. Synergy between nanomaterials and volatile organic compounds for non-invasive medical evaluation. Chem Soc Rev 2018; 47: 4781-4859.

42 Kim HJ, Lee JH. Highly sensitive and selective gas sensors using p-type oxide semiconductors: overview. Sens Actuators B Chem 2014; 192: 607-627. 
43 Piriya VSA, Joseph P, Daniel SCGK, et al. Colorimetric sensors for rapid detection of various analytes. Mater Sci Eng C Mater Biol Appl 2017; 78: 1231-1245.

44 Jakubik WP. Surface acoustic wave-based gas sensors. Thin Solid Films 2011; 520: 986-993.

45 Nakhleh MK, Broza YY, Haick H. Monolayer-capped gold nanoparticles for disease detection from breath. Nanomedicine 2014; 9: 1991-2002.

46 Peled N, Barash O, Tisch U, et al. Volatile fingerprints of cancer specific genetic mutations. Nanomedicine 2013; 9: 758-766.

47 Barash O, Peled N, Hirsch FR, et al. Sniffing the unique "odor print" of non-small-cell lung cancer with gold nanoparticles. Small 2009; 5: 2618-2624.

48 Barash O, Peled N, Tisch U, et al. Classification of lung cancer histology by gold nanoparticle sensors. Nanomedicine 2012; 8: 580-589.

49 Filipiak W, Sponring A, Filipiak A, et al. TD-GC-MS analysis of volatile metabolites of human lung cancer and normal cells in vitro. Cancer Epidemiol Biomarkers Prev 2010; 19: 182-195.

50 Sponring A, Filipiak W, Ager C, et al. Analysis of volatile organic compounds (VOCs) in the headspace of NCI-H1666 lung cancer cells. Cancer Biomark 2010; 7: 153-161.

51 Jia Z, Zhang $\mathrm{H}$, Ong $\mathrm{CN}$, et al. Detection of lung cancer: concomitant volatile organic compounds and metabolomic profiling of six cancer cell lines of different histological origins. ACS Omega 2018; 3: 5131-5140.

52 Horváth I, Barnes PJ, Loukides S, et al. A European Respiratory Society technical standard: exhaled biomarkers in lung disease. Eur Respir J 2017; 49: 1600965.

53 Konvalina G, Haick H. Sensors for breath testing: from nanomaterials to comprehensive disease detection. Acc Chem Res 2014; 47: 66-76.

54 Broadhurst DI, Kell DB. Statistical strategies for avoiding false discoveries in metabolomics and related experiments. Metabolomics 2006; 2: 171-196.

55 Kort S, Tiggeloven MM, Brusse-Keizer M, et al. Multi-centre prospective study on diagnosing subtypes of lung cancer by exhaled-breath analysis. Lung Cancer 2018; 125: 223-229.

56 Mazzone PJ. Analysis of volatile organic compounds in the exhaled breath for the diagnosis of lung cancer. J Thorac Oncol 2008; 3: 774-780.

57 Collins GS, Reitsma JB, Altman DG, et al. Transparent reporting of a multivariable prediction model for individual prognosis or diagnosis (TRIPOD): the TRIPOD statement. BMC Med 2015; 13: 1.

58 Cohen JF, Korevaar DA, Altman DG, et al. STARD 2015 guidelines for reporting diagnostic accuracy studies: explanation and elaboration. BMJ Open 2016; 6: e012799. 